\title{
Utilisation d'un site francophone de données probantes pharmaceutiques dans la formation universitaire et continue des pharmaciens
}

\author{
Usefulness of a website on pharmacy evidence in university training \\ and pharmacist continuing education
}

\author{
Aurélie GUÉRIN ${ }^{1}$, Denis LEBEL ${ }^{2}$, Jean-François BUSSIÈRES ${ }^{3}$ \\ 1 Unité de recherche en pratique pharmaceutique, CHU Sainte-Justine, Montréal, Québec, Canada \\ 2 Département de Pharmacie, Unité de recherche en pratique pharmaceutique, CHU Sainte-Justine, Montréal, Québec, \\ Canada \\ 3 Département de Pharmacie, Unité de recherche en pratique pharmaceutique, CHU Sainte-Justine, Montréal, Québec, \\ Faculté de Pharmacie, Université de Montréal, Montréal, Québec, Canada
}

Manuscrit reçu le 17 septembre 2014 ; commentaires éditoriaux formulés aux auteurs le 7 et 16 mars 2015 ; accepté pour publication le 20 mars 2015

\begin{abstract}
Mots-clés
Pratique

pharmaceutique ;

formation

universitaire ;

formation continue

données probantes ;

francophonie

Résumé - Contexte : Il existe peu de place accordée aux résultats issus de la recherche évaluative sur les pratiques professionnelles dans la formation des pharmaciens. Méthodes : Nous avons développé une démarche structurée de revue et d'analyse documentaire des données probantes disponibles, associée à une stratégie de transfert de connaissances incluant la création d'un site Internet francophone, le site Impact Pharmacie. Sur ce site, 70 fiches de synthèse relatives à des affections, des programmes de soins ou des activités pharmaceutiques présentent l'implication des pharmaciens. On retrouve au sein de ces fiches toutes les activités décrites dans la littérature ainsi que les retombées de ces activités sur plusieurs marqueurs, tel que la morbidité et les coûts. Résultats : Lors d'une conférence d'information incluant la présentation d'articles pivots sur le rôle et les retombées du pharmacien et la présentation du site Internet Impact Pharmacie, nous avons évalué l'exposition et la perception de pharmaciens québécois vis à vis des données probantes en pratique pharmaceutique. Les participants soutenaient l'utilisation de cette littérature et affirmaient qu'ils n'y étaient pas suffisamment exposés. Les répondants considéraient le site Impact Pharmacie clair, pertinent, devant être intégré au cursus universitaire et utile à la pratique professionnelle. Conclusion : Nous pensons que le site
\end{abstract}


Impact Pharmacie est un outil pédagogique innovant didactique qui peut être utile à la réflexion des pharmaciens dans la francophonie, d'où le partage de ces résultats et de cette approche de sensibilisation.

\section{Keywords}

Pharmacy practice; university curriculum; continuing education; evidence-based; French-speaking community

\begin{abstract}
Background: In our view, there is too little emphasis on the results stemming from evaluative research on professional practice in pharmacist training. Methods: We developed a structured approach that included a literature review of evidence, combined with a strategy of knowledge transfer including the creation of the French-language Impact Pharmacie website. On the website, fact sheet pages present an overview of the roles of pharmacists on a given topic, including a description of activities and its impact on multiple outcomes, including morbidity and costs. Results: During an information conference that included the presentation of relevant articles on the role and impact of pharmacists and the presentation of the Impact Pharmacie website, we evaluated Quebec pharmacists' exposure to and perception of pharmacy practice evidence. The participants supported the use of the literature and stated that they were not sufficiently exposed to the evidence. Respondents thought the Impact Pharmacie website was clear and relevant, felt that it should be incorporated in the academic curriculum and found it useful in their professional practice. Conclusion: We believe that the Impact Pharmacie website is an innovative educational tool that can provide food for thought to pharmacists, which is why the results and the awareness-raising approach are being shared.
\end{abstract}

\section{Introduction}

Alors que les pharmaciens sont exposés aux meilleures données probantes portant sur la pharmacothérapie au cours de leur cursus universitaire, nous savons peu de choses sur leur exposition à la recherche évaluative sur les pratiques professionnelles. À notre avis, peu de place est accordée aux résultats issus de la recherche évaluative sur les pratiques professionnelles dans la formation des pharmaciens. Ce déficit prépare mal les pharmaciens à s'intéresser à la recherche évaluative, et à y contribuer activement durant leur carrière ou à intégrer les résultats de ces études dans leur pratique.

Un tel constat met en évidence l'importance de trouver des moyens de formation continue qui exposent plus systématiquement les pharmaciens aux données probantes afin qu'ils prennent des décisions éclairées face aux nombreux changements de la pratique pharmaceutique. Ainsi, il apparaît opportun d'intégrer les meilleures données relatives au rôle et aux retombées des pharmaciens dans leur formation universitaire et continue.
Cependant peu de ressources ou d'outils mettent en valeur les meilleures données probantes de l'activité pharmaceutique. En Europe, on retrouve sur le site de l'association européenne des pharmaciens hospitaliers, une revue de littérature statique qui contient 136 articles sur les retombées de l'activité pharmaceutique $^{[1]}$. On identifie aussi une initiative danoise datant de 2001, destinée seulement aux pharmaciens d'officine ${ }^{[2]}$, en remarquant cependant une absence de mise à jour récente de cette base de données.

En France, dans un état de lieux de la pharmacie, Calop et al. ont mentionné en 2009 que « nous disposons de très peu de données décrivant les pratiques de nos collègues » ${ }^{[3]}$.

La Société Française de Pharmacie Clinique a mis en place en novembre 2004 un outil baptisé « ACT IP» permettant notamment l'enseignement de la pharmacie clinique ${ }^{[4]}$.

Consciente à la fois de la faible exposition des pharmaciens aux données probantes récentes concernant le rôle et les retombées du pharmacien, et de l'absence de ressources pédagogiques, l'équipe de l'Unité de recherche en pratique pharmaceutique du 
CHU Sainte Justine (URPP) s'est intéressé à ces données probantes.

\section{Développement d'un site Internet francophone présentant les données probantes descriptives et les retombées des activités du pharmacien}

Le projet de recherche a pris la forme d'une démarche structurée de revue et d'analyse documentaire des données probantes disponibles, associée à une stratégie de transfert de connaissances incluant la création d'un site Internet francophone ${ }^{[5]}$.

Une méthode reproductible a été élaborée afin de recueillir, analyser et présenter les données probantes relatives aux rôles et aux retombées de l'activité pharmaceutique.

La méthode comportait cinq principales étapes, soit le choix des thèmes, la recherche bibliographique, la rédaction de fiche par article, la rédaction de fiches synthèses par thème et le transfert des données sur un site Internet. Le projet a reposé sur la contribution scientifique de quinze assistants de recherche formés selon une méthode structurée, avec un assistant de recherche pivot sous la gouverne de deux pharmaciens de l'équipe de recherche. Toutes les fiches synthèses ont été relues par trois membres de l'équipe de recherche.

La démarche structurée et reproductible ${ }^{[6]}$ a mené à l'inclusion de 1442 articles sur le site Internet, dont 914 ont été analysés en détail en date du 20 août 2014. À partir des articles inclus, 70 fiches de synthèse ont été rédigées selon différents thèmes, réparties en 19 affections (par exemple, insuffisance cardiaque), 28 programmes de soins hospitalisés ou ambulatoires (par exemple, gériatrie) et 23 activités pharmaceutiques (par exemple, éducation thérapeutique/conseils aux patients).

L'onglet «Données » du site Internet Impact Pharmacie permet une exploration du rôle et des retombées de l'activité pharmaceutique par affections, activités pharmaceutiques, programmes de soins et provenances. L'affichage des données est effectué selon une taille proportionnelle à leur poids relatif sur le site, à l'aide d'une visualisation interactive. Par exemple, la consultation des données relatives aux affections met en évidence la quantité plus grande de preuves sur le thème du diabète que sur d'autres affections recensées dans le site. Les articles sont classés par ordre décroissant de date de réalisation de l'étude. Un lien vers l'analyse de chaque article et la source Pubmed sont disponibles.

Les fiches de synthèse présentent un résumé de l'implication des pharmaciens dans un domaine particulier, incluant les activités décrites dans la littérature ainsi que les retombées de ces activités sur plusieurs marqueurs, tel que la morbidité et les coûts.

L'onglet «Synthèse » du site Internet Impact Pharmacie permet un accès aux 70 fiches synthèses classées par ordre alphabétique. Mentionnons que la navigation au sein d'une fiche synthèse se fait en « entonnoir ». Ainsi le lecteur intéressé par le diabète peut cliquer sur la fiche de synthèse et $\mathrm{y}$ trouver notamment l'ensemble des indicateurs de retombées et des indicateurs descriptifs du rôle du pharmacien. Le lecteur intéressé par un indicateur ou un milieu de pratique peut cliquer sur les liens pour obtenir l'analyse de l'article. Enfin, le lecteur peut avoir accès au résumé PubMed s'il s'agit d'un article indexé sur PubMed et à l'article source si celui-ci est disponible.

Le site Internet francophone http://impactpharmacie.org est ouvert à tous les lecteurs intéressés, sans inscription nécessaire. À la différence de plusieurs sites de données statiques sans mise à jour périodique, le site Internet Impact Pharmacie fera l'objet d'une mise à jour continuelle. Cette mise à jour inclura une collaboration avec des pharmaciens experts pour chaque thématique. De plus, notre équipe de recherche commente chaque semaine un article récent à propos de l'activité du pharmacien sur un blogue ${ }^{[7]}$.

\section{Utilisation lors d'une activité de formation continue au Québec}

Nous avons animé trois ateliers identiques « Intervenir avec Impact » lors du congrès annuel de l'Ordre des pharmaciens du Québec, tenu à Montréal le 17 et 18 juin $2014^{[8]}$. Cet atelier interactif de 90 minutes 
discutait de l'importance de la recherche en pratique pharmaceutique, des forces et des faiblesses de cette discipline, la présentation d'articles pivots sur le rôle et les retombées du pharmacien et la présentation du site Internet Impact Pharmacie. Cet atelier informatif visait à faire connaitre le site Impact Pharmacie et ses modalités d'utilisation.

Lors de ces ateliers, nous avons développé un questionnaire visant à explorer la perception des pharmaciens vis-à-vis des données probantes en pratique pharmaceutique et leurs premières impressions du site Impact Pharmacie. Le questionnaire a été développé et pré-testé avec la collaboration de pharmaciens. Il a été administré à la fin de l' atelier à un échantillon de participants volontaires. Ce questionnaire comportait 31 questions en version papier. Pour chaque énoncé, les participants étaient invités à coter leur niveau d'accord sur une échelle de 0 à 10 (0-totalement en désaccord, 10 - totalement en accord). Bien qu'il s'agisse d'une échelle ordinale, et en accord avec certains arguments ${ }^{[9]}$, une moyenne arithmétique du niveau d'accord a été calculée pour chacun des énoncés. Une valeur supérieure à 7 est associée à un niveau élevé d'appui des pharmaciens à l'énoncé.

Au total, 70 pharmaciens ont participé au sondage (100\% des participants à l'atelier). Soixantequatre pourcent $(n=45)$ des répondants étaient des femmes et $74 \%(n=52)$ exerçaient en officine. Les répondants avaient en moyenne 15 années d'expérience en pratique pharmaceutique. Trente-neuf pourcent $(n=27)$ avaient entre 41 et 50 ans, $23 \%$ $(n=16)$ entre 31 et 40 ans, $19 \%(n=13)$ entre 51 et 60 ans, $17 \%(n=12)$ moins de 30 ans et $2 \%(n=1)$ plus de 60 ans.

Moins de $6 \%$ des répondants n'avaient lu aucun article portant sur la pharmacothérapie au cours des 12 derniers mois. Toutefois, près de $34 \%$ n'avaient lu aucun article portant sur les modèles de pratique dans les 12 derniers mois, et seulement $11 \%$ des participants ont lu plus de 10 articles par année. Ainsi, l'exposition aux données probantes sur le rôle et les retombées du pharmacien semble plus limitée que celle portant sur la pharmacothérapie. Néanmoins, on note que les participants soutiennent l'utilisation des meilleures données probantes sur les pratiques professionnelles. Ils considèrent qu'ils y sont insuffisamment exposés, mais envisagent de les intégrer dans leur pratique (tableau I).

En tenant compte des données recueillies, nous notons que les pharmaciens appuient le fait que «l'exposition d'un pharmacien à des données probantes scientifiques sur les pratiques professionnelles (par exemple, un nouvel article démontrant la capacité du pharmacien à améliorer significativement le ratio HDL/LDL) amène des changements de pratique ». Ainsi, le site Impact Pharmacie est susceptible de contribuer à des changements de pratique au fil du temps. De même, les pharmaciens sont d'avis que les données probantes scientifiques relatives aux retombées de l'exercice de la pharmacie sont utiles pour les exposer au cadre législatif, à la gestion et à la révision des pratiques. Si la formation universitaire proposée au Québec pour la formation d'un pharmacien expose les étudiants suffisamment aux données probantes relatives à la pharmacothérapie (score moyen de 7,8), cette exposition apparaît insuffisante en ce qui concerne l'évaluation des pratiques professionnelles (score moyen de 5,3).

Suite à la présentation du site Impact Pharmacie, lors des ateliers, les répondants ont considéré que le site était clair et pertinent, qu'il devrait être intégré au cursus universitaire et qu'il est utile à la pratique professionnelle (tableau II). L'ensemble de ces données bien que n'ayant pas fait l'objet d'une analyse statistique, mettent en évidence un besoin patent d'exposer davantage les pharmaciens aux données probantes concernant leur rôle et de leurs retombées.

\section{Utilisation du site Internet dans les curriculums en pharmacie}

Le site Internet constitue un outil pédagogique innovant didactique, regroupant et mettant à disposition les données sur le rôle et les retombées du pharmacien dans un site facilement accessible. La mise à jour annuelle du site et de son blogue hebdomadaire permet de partager périodiquement des nouvelles d'intérêt. Le site peut contribuer à l'enseignement de l'évaluation des pratiques professionnelles dès le premier cycle des cursus universitaires de pharmacie. 
Tableau I. Perceptions des pharmaciens participant à l'atelier vis-à-vis des données probantes en pratique pharmaceutique.

\begin{tabular}{|c|c|}
\hline Énoncés & $\begin{array}{l}\text { Niveau d'accord moyen des } \\
\text { pharmaciens }(n=70)\end{array}$ \\
\hline $\begin{array}{l}\text { Je pense que l'exposition d'un patient à des preuves scientifiques } \\
\text { (p.ex. taux de cancer du poumon associé au tabagisme) amène des changements } \\
\text { d'attitude }\end{array}$ & 6,6 \\
\hline $\begin{array}{l}\text { Je pense que l'exposition d'un pharmacien à des preuves scientifiques } \\
\text { sur le médicament (p.ex. un nouvel article sur l'efficacité d'une statine) amène des } \\
\text { changements de pratique }\end{array}$ & 8,3 \\
\hline $\begin{array}{l}\text { Je pense que l'exposition d'un pharmacien à des preuves scientifiques } \\
\text { sur les pratiques professionnelles (p.ex. un nouvel article démontrant la capacité } \\
\text { du pharmacien à améliorer significativement le ratio HDL/LDL) amène } \\
\text { des changements de pratique }\end{array}$ & 7,9 \\
\hline $\begin{array}{l}\text { Je pense que les preuves scientifiques relatives aux retombées de l'exercice } \\
\text { de la pharmacie sont utiles pour la décision du législateur afin d'élargir l'exercice } \\
\text { de la pharmacie }\end{array}$ & 8,0 \\
\hline $\begin{array}{l}\text { Je pense que les preuves scientifiques relatives aux retombées de l'exercice de la } \\
\text { pharmacie sont utiles pour la décision de gestionnaires pour financer des services } \\
\text { pharmaceutiques }\end{array}$ & 7,5 \\
\hline $\begin{array}{l}\text { Je pense que les preuves scientifiques relatives aux retombées de l'exercice } \\
\text { de la pharmacie sont utiles pour les décisions de remboursement des services } \\
\text { et activités cognitives pharmaceutiques }\end{array}$ & 7,5 \\
\hline $\begin{array}{l}\text { Je pense que les preuves scientifiques relatives aux retombées de l'exercice } \\
\text { de la pharmacie sont utiles pour la révision des pratiques des pharmaciens }\end{array}$ & 8,3 \\
\hline $\begin{array}{l}\text { Je pense que les preuves scientifiques relatives aux retombées de l'exercice } \\
\text { de la pharmacie sont utiles pour influencer positivement la perception des autres } \\
\text { professionnels vis-à-vis des pharmaciens }\end{array}$ & 7,9 \\
\hline $\begin{array}{l}\text { Je pense que les preuves scientifiques relatives aux retombées de l'exercice } \\
\text { de la pharmacie sont utiles pour influencer positivement la perception des patients } \\
\text { du rôle et de la portée de l'exercice de la pharmacie }\end{array}$ & 6,8 \\
\hline $\begin{array}{l}\text { Durant ma formation à l'université (incluant les stages), mes enseignements } \\
\text { relatifs à la pharmacothérapie incluaient systématiquement des preuves } \\
\text { scientifiques }\end{array}$ & 7,8 \\
\hline $\begin{array}{l}\text { Durant ma formation à l'université (incluant les stages), mes enseignements } \\
\text { relatifs à l'évaluation des pratiques professionnelles incluaient systématiquement } \\
\text { des preuves scientifiques }\end{array}$ & 5,3 \\
\hline $\begin{array}{l}\text { Je suis capable de trouver des preuves scientifiques pertinentes pour répondre } \\
\text { à mes questions pratiques }\end{array}$ & 7,3 \\
\hline $\begin{array}{l}\text { Je suis capable de réaliser des lectures critiques d'articles (p.ex. identifier } \\
\text { l'objectif, analyser la méthodologie, analyser les résultats, évaluer l'applicabilité) } \\
\text { pour répondre à mes questions pratiques }\end{array}$ & 6,7 \\
\hline $\begin{array}{l}\text { J'intègre les données des preuves scientifiques dans ma pratique relative } \\
\text { à la pharmacothérapie }\end{array}$ & 7,3 \\
\hline $\begin{array}{l}\text { J'intègre les données des preuves scientifiques dans ma pratique relative } \\
\text { à l'évaluation des pratiques }\end{array}$ & 6,5 \\
\hline
\end{tabular}

Échelle : 0 : parfaitement en désaccord et 10 : parfaitement en accord. 
Tableau II. Perception des pharmaciens participant à l'atelier vis-à-vis du site Impact Pharmacie après sa présentation.

\begin{tabular}{|l|c|}
\hline Énoncés & $\begin{array}{c}\text { Niveau d'accord moyen } \\
\text { des pharmaciens }(\boldsymbol{n}=\mathbf{7 0})\end{array}$ \\
\hline Le contenu du site Impact Pharmacie est présenté clairement & 8,4 \\
\hline Le contenu du site Impact Pharmacie est pertinent & 8,8 \\
\hline Le contenu du site Impact Pharmacie devrait être intégré au cursus du Pharm.D & 8,8 \\
\hline $\begin{array}{l}\text { Le contenu du site Impact Pharmacie devrait être intégré au cursus de la maitrise } \\
\text { en pharmacothérapie avancée }\end{array}$ & 9,0 \\
\hline $\begin{array}{l}\text { Le contenu du site Impact Pharmacie devrait être utilisé en gestion afin d'informer } \\
\text { les décideurs en santé }\end{array}$ & 9,1 \\
\hline Le contenu du site Impact Pharmacie peut être utile à ma pratique personnelle & 8,1 \\
\hline
\end{tabular}

Échelle : 0 : parfaitement en désaccord et 10 : parfaitement en accord.

Les données probantes sur les retombées du pharmacien devraient être intégrées aux approches pédagogiques en place pour l'enseignement. Ainsi lors de l'enseignement d'un thème donné (par exemple, l'hypertension, la gériatrie), l'enseignant peut proposer aux élèves la lecture de la fiche de synthèse associée. Les élèves peuvent ainsi être exposés aux interventions réalisées par les pharmaciens dans leur milieu de travail, et constater celles qui ont des retombées réelles sur les patients.

La recherche évaluative devrait également être valorisée et enseignée dans les programmes de deuxième et troisième cycles afin que les étudiants reconnaissent les meilleures pratiques et puissent les implanter en début de carrière. La connaissance de cette vaste littérature sur l'évaluation des pratiques peut également encourager les jeunes pharmaciens à remettre en question leurs activités et à évaluer leurs retombées. On peut consulter trois démarches suggérées visant à faciliter l'utilisation du site Impact Pharmacie et de son blogue auprès des cliniciens, des décideurs en santé et des enseignants $[10,11,12]$.

\section{Utilisation du site Internet dans la formation continue et l'amélioration des pratiques des pharmaciens}

Exercer la pharmacie en 2015, repose sur les données probantes. En France, la Haute Autorité de Santé
(HAS) définit l'évaluation des pratiques professionnelles comme l'analyse de l'activité d'un professionnel de santé réalisée par rapport aux recommandations professionnelles disponibles actualisées, afin de mettre en œuvre un plan d'amélioration de son activité professionnelle et de la qualité des soins délivrés aux patients ${ }^{[13]}$.

Nous pensons que le site Impact Pharmacie s'inscrit dans cette démarche d'évaluation des pratiques professionnelles, en constituant une source de données probantes professionnelles disponibles et actualisées. Par ailleurs, notons que la loi « Hôpital, patients, santé et territoires » (HPST) (Loi n²009879 du 21 juillet 2009 portant réforme de l'hôpital et relative aux patients, à la santé et aux territoires) instaure l'obligation de développement professionnel continu (DPC) pour l'ensemble des professionnels de santé en France. Selon l'article 59 de cette loi, le DPC a notamment pour objectifs l'évaluation des pratiques professionnelles ${ }^{[14,15]}$. Nous pensons que le site Impact Pharmacie pourrait s'intégrer dans cette démarche visant à l'amélioration des pratiques professionnelles.

\section{Conclusion}

Nous pensons que le site Impact Pharmacie est un outil innovant de ressources didactiques, qui peut être utile à la réflexion des pharmaciens dans la francophonie, d'où le partage de ces résultats et de cette approche de sensibilisation aux données probantes. 


\section{Remerciements}

Nous remercions le Ministère de la santé et des services sociaux du Québec pour un financement sans restriction pour le développement initial du site Impact Pharmacie.

\section{Valorisation scientifique}

Une partie du travail a été présentée dans le cadre du Colloque du Réseau québécois de recherche sur l'usage des médicaments, les 22-23 septembre 2014 à Orford, Québec, Canada.

\section{Contributions}

Aurélie Guérin a contribué à la méthodologie de recherche, aux recherches bibliographiques, à l'analyse des d'articles, aux saisies dans la base de données, à la rédaction des fiches de synthèse et aux publications sur le site Internet. Elle a contribué à l'élaboration des questions du sondage, à l'analyse des données, à la rédaction du manuscrit, à la révision scientifique du contenu et à l'approbation de la version finale. Denis Lebel et Jean-François Bussières ont contribué à l'identification de la problématique, à la méthodologie de recherche, à la conception du site Internet. Ils ont contribué à l'élaboration des questions du sondage, à la révision scientifique du contenu et à l'approbation de la version finale.

\section{Déclaration d'intérêts}

Les auteurs ne déclarent aucun conflit d'intérêt en lien avec le contenu de cet article.

\section{Approbation éthique}

Sans objet

\section{Références}

1. European Association of hospital pharmacists. Hospital pharmacy. [On-line]. Disponible sur: http://www.eahp.eu/practice-and-policy/hospitalpharmacy

2. Pharmakon. The Danish community pharmacy evidence database. [On-line]. Disponible sur : http://www.pharmakon.dk/pages/ international. aspx?pageid=149

3. Calop J, Baudrant M, Bedouch P, Allenet B. La pharmacie clinique en France : contexte de développement à l'hôpital et état des lieux. Pharmactuel 2009;42:34-39.

4. Société française de pharmacie clinique. Standardisation et valorisation des activités de pharmacie clinique - Outil ACT IP. [On-line]. Disponible sur : http://sfpc.eu/fr/groupes-travail/interventionspharmaceutiques.html

5. Unité de recherche en pratique pharmaceutique. Impact Pharmacie [On-line]. Disponible sur : http://impactpharmacie.org/

6. Guérin A, Barthélémy I, Merger D, Tanguay C, Lebel $\mathrm{D}$, Bussières JF. Projet Impact Pharmacie sur la description du rôle et sur les retombées du pharmacien : méthodologie. Annales de l'Unité de recherche en pratique pharmaceutique. 2014 [Online]. Disponible sur : http://indicible.ca/urpp/ 20140130_IMPACTPHARMACIE2_Annales.pdf

7. Unité de recherche en pratique pharmaceutique. Le pharmacien est incontournable [On-line]. Disponible sur : http://impactpharmacie.wordpress.com/ a-propos/

8. Bussières JF, Guérin A. Atelier : Intervenir avec impact. Experts en santé, se donner les moyens d'avancer. Colloque de l'Ordre des pharmaciens du Québec, 17-18 juin 2014. Montréal (QC), Canada.

9. Carifio J, Perla R. Resolving the 50-year debate around using and misusing Likert scales. Med Educ 2008;42:1150

10. Guérin A, Lebel D, Ferreira E, Bédard P, Bussières JF. Projet Impact Pharmacie : utilisation par les pharmaciens. Annales de l'Unité de recherche en pratique pharmaceutique 2014. [On-line]. Disponible sur : http://indicible.ca/urpp/ 20140828_IMPACTPHARMACIE9_Annales.pdf 
11. Guérin A, Lebel D, Ferreira E, Bédard P, Bussières JF. Projet Impact Pharmacie : utilisation par les décideurs en santé. Annales de l'Unité de recherche en pratique pharmaceutique. 2014 [On-line]. Disponible sur: http://indicible.ca/urpp/20140828_ IMPACTPHARMACIE10_Annales.pdf

12. Guérin A, Lebel D, Ferreira E, Bédard P, Bussières JF. Projet Impact Pharmacie : utilisation en pédagogie pharmaceutique. Annales de l'Unité de recherche en pratique pharmaceutique 2014 [Online]. Disponible sur: http://indicible.ca/urpp/ 20140828_IMPACTPHARMACIE11_Annales.pdf

13. Haute Autorité de Santé. Évaluation des pratiques professionnelles. [On-line]. Disponible sur http:// www.has-sante.fr/portail/jcms/c_711453/fr/evaluation-des-pratiques-professionnelles?xtmc $=\& x t c r=1$
14. Haute Autorité de Santé. Cadre légal et historique du développement professionnel continue. [On-line]. Disponible sur : http://www.has-sante.fr/portail/ jcms/c_1288567/fr/cadre-legal-et-historique-du$\mathrm{dpc}$

15. Code de la santé publique. Chapitre III Développement professionnel continu. [On-line]. Disponible sur: http://www.legifrance.gouv.fr/affichCode.do;jsessionid=2093207095A23DA8CF9944BC9A018BE0.tpdjo16 v_3?idSectionTA=LEGISCTA000020897546\&cidTexte= LEGITEXT000006072665\&dateTexte $=20100310$

Correspondance et offprints : Jean-François Bussières. Département de Pharmacie, Unité de recherche en pratique pharmaceutique, CHU Sainte-Justine, 3175 chemin Côte-Ste-Catherine, H3T1C5 Montréal (Québec), Canada.

Mailto : jf.bussieres@ssss.gouv.qc.ca 